\title{
BMJ Open Quality Improving TB infection control in a regional hospital in the Eastern Cape, South Africa
}

\author{
Ilsa Louisa Haeusler, ${ }^{\oplus 1}$ Felicity Knights, ${ }^{1}$ Vishaal George, ${ }^{2}$ Andy Parrish ${ }^{1,3,4}$
}

To cite: Haeusler IL, Knights F, George V, et al. Improving TB infection control in a regional hospital in the Eastern Cape, South Africa. BMJ Open Quality 2019;8:e000347. doi:10.1136/ bmjoq-2018-000347

- Additional material is published online only. To view please visit the journal online (http://dx.doi.org/10.1136/ bmjoq-2018-000347).

Received 29 January 2018 Revised 12 September 2018 Accepted 25 January 2019

Check for updates

(c) Author(s) (or their employer(s)) 2019. Re-use permitted under CC BY-NC. No commercial re-use. See rights and permissions. Published by BMJ.

${ }^{1}$ Improving Global Health through Leadership Development, NHS Thames Valley and Wessex Leadership Academy, Otterbourne, UK ${ }^{2}$ Department of Physiotherapy, Cecilia Makiwane Hospital,

Mdantsane, Eastern Cape, South Africa

${ }^{3}$ Department of Internal Medicine, Walter Sisulu University School of Medicine, Mthatha, Eastern Cape, South Africa

${ }^{4}$ Department of Internal Medicine, Cecilia Makiwane Hospital, Mdantsane, Eastern Cape, South Africa

Correspondence to Dr Ilsa Louisa Haeusler; ilsa.haeusler@gmail.com

\section{ABSTRACT}

This quality improvement (QI) work was carried out in Cecilia Makiwane Hospital (CMH), a regional public hospital in the Eastern Cape, South Africa (SA). SA has among the highest incidence of tuberculosis (TB) in the world and this is a leading cause of death in SA. Nosocomial infection is an important source of TB transmission. Adherence to TB infection prevention control (IPC) measures in the medical inpatient department was suboptimal at $\mathrm{CMH}$. The overall aim of this QI project was to make sustainable improvements in TB IPC. A multidisciplinary team was formed to undertake a root cause analysis and develop a strategy for change. The main barriers to adherence to IPC measures were limited knowledge of IPC methods and stigma associated with TB. Specifically, the project aimed to increase the number of: 'airborne precaution' signs placed above patients' beds, patients correctly isolated and patients wearing surgical face masks. Four PlanDo-Study-Act cycles were used. The strategy for change involved education and awareness-raising in different formats, including formal in-service training delivered to nurses and doctors, a hospital-wide TB awareness week with engaging activities and competitions, and a World TB Day provincial solidarity march. Data on adherence to the three IPC measures were collected over an 8-month period. Pre-intervention (October 2016), a mean of $2 \%$ of patients wore face masks, $22 \%$ were correctly isolated and $12 \%$ had an airborne precaution sign. Postintervention (May 2017), the compliance improved to $17 \%$, $50 \%$ and $25 \%$, respectively. There was a large variation in compliance to each measure. Improvement was greatest in the number of patients correctly isolated. We learnt it is important to work with, not in parallel to, existing teams or structures during QI work. On-the-ground training of nurses and clinicians should be undertaken alongside engagement of senior staff members and managers. This improves the chance of change being adopted into hospital policy.

\section{PROBLEM}

The incidence of tuberculosis (TB) in South Africa (SA) is 834 per 100000 , which is among the highest in the world, ${ }^{1}$ and the disease is a leading cause of death. ${ }^{2}$ TB in SA is associated with high rates of drug resistance, and up to $60 \%$ of patients with TB are HIV coinfected. ${ }^{3}$ In 2015 there were 19613 multidrug-resistant cases and 1024 extensively drug-resistant cases in SA, second only to India. ${ }^{1}$ The $2 \%$ incidence of TB among healthcare workers in SA is twice that of the general population, suggesting nosocomial transmission. ${ }^{4}$ Globally, the WHO's 'End TB Strategy' called for a $95 \%$ reduction in the number of $\mathrm{TB}$ deaths and a $90 \%$ reduction in $\mathrm{TB}$ incidence rate by $2035 .^{5}$

This work was carried out in Cecilia Makiwane Hospital $(\mathrm{CMH})$, which is a regional public hospital in the Eastern Cape, SA, with 1246 staff. CMH is situated in Mdantsane, a township with a population of $157000 .^{6}$ The Eastern Cape, where life expectancy is 54 years for men and 59 for women, ${ }^{7}$ is the province with the second largest number of annual TB deaths in SA, surpassed by the neighbouring province of KwaZulu-Natal. ${ }^{2}$

Despite the large number of potentially infectious patients with $\mathrm{TB}$ presenting to $\mathrm{CMH}$, adherence to infection control practices was felt by clinicians to be suboptimal, posing a risk to their own health and that of other patients. This work aimed to improve adherence to TB infection prevention control (IPC) practices over a 3-month period on the acute medical inpatient wards at $\mathrm{CMH}$ by a $25 \%$ increase in each of the following:

- The use of 'Airborne Precaution' signs above the beds of patients with symptoms suggestive of TB.

- The use of surgical face masks by patients with symptoms suggestive of TB.

- The number of infectious patients in whom isolation measures were attempted. Following the first 3-month period, the project continued with the aim of maintaining improvement in each of these areas over a further 5 -month period.

\section{BACKGROUND}

Nosocomial transmission of TB is a growing concern in SA. ${ }^{8}$ TB IPC measures are effective at reducing transmission. The $\mathrm{WHO}$ and the Centres for Disease Control have published guidelines on IPC interventions for use in healthcare settings, ${ }^{9} 10$ and the 
National South African TB IPC guidelines are aligned with these recommendations. ${ }^{11}$

The WHO categorises TB IPC into work practice, administrative and environmental controls. Work practice aims to prevent infectious droplet nuclei from entering the environment by ensuring rapid diagnosis and management of people with suspected or known TB. This involves prompt screening of patients entering healthcare facilities to identify potential patients with TB, providing surgical face masks to patients (respiratory hygiene) and separation of potential patients with TB from other patients (isolation). Administrative processes include establishment of an institutional IPC committee and development of a hospital TB IPC plan, which incorporates staff training. Environmental controls are a second line of defence for preventing spread of infection, and include natural and mechanical ventilation, ultraviolet germicidal light and the use of N95 respirator masks (personal respiratory protection).

A major barrier to implementing TB IPC practices at $\mathrm{CMH}$ was the staffs' incomplete knowledge of specific TB IPC practices. This included a limited understanding of which patients are infectious and which patients require TB IPC measures. There was limited knowledge of isolation practices, in particular alternative techniques to use when isolation rooms are not available, and how and when to apply respiratory protection (surgical and N95 face masks). A further barrier was the stigma associated with TB. This particularly affected staffs' willingness to ensure infectious patients were nursed away from other patients and to ask patients to wear surgical face masks during the infectious stage of the illness.

The hospital has an inadequate number of isolation rooms, no artificial ventilation, limited natural ventilation and no artificial ultraviolet lighting. Resource limitations extended to the supply of N95 respirators, and when supplied they were often locked away, out of access of members of staff.

Staff members at CMH were aware of these environmental limitations and were worried about how these limitations affected their risk of TB infection. They were less aware of how they could optimise the system and environment to reduce risk. These concerns and attitudes are not unique to this facility; similar concerns were found in a qualitative study of nurses' attitudes regarding TB IPC in the Western Cape Province in SA. ${ }^{12}$

\section{MEASUREMENT}

The overall aim was to reduce nosocomial transmission of TB. As an outcome measure, it is difficult to measure the number of hospital-acquired TB infections because it would take years to collect this information and it can be challenging to prove causality. Instead we chose three process measures. The team felt it was important for these measures to be in line with national policy so that a consistent and evidence-based approach could be adopted.
The first process measure was the number of infectious patients where isolation had been attempted. This was defined as placing a patient in an isolation bay, or separating from other patients by placement at the end of a bay or in an area with surrounding empty beds. This outcome measure allowed for the structural constraints within the facility by making use of alternative methods to isolate patients.

The second measure was the number of infectious patients who had an 'airborne precaution' sign positioned above the bed or on the door, indicating that airborne precaution personal protective equipment should be worn when working with the patient. The rationale behind this intervention was that members of the staff, including doctors, nurses, therapists and housekeepers, were often unaware of a patient's infectiousness and this thus established a means to identify patients who are potentially infectious.

The third measure was the number of infectious patients who were wearing face masks correctly (covering their nose and mouth), or those who had face masks around their face or neck or within easy reach of their bed. This less stringent definition was chosen because it is unlikely that a patient will be able to keep a face mask on at all times, for example when eating, and that the presence of a face mask near a patient would indicate a significant improvement from the baseline practice.

For each of these process measures, we included as the denominator any patient who required IPC measures, that is, any patient who was infectious (confirmed pulmonary TB treated for less than 2 weeks) or any patient who was potentially infectious (a patient with a high clinical index of suspicion of TB but Gene Xpert results pending). These diagnoses were made by the medical doctors (interns and registrars supported by consultant colleagues) and communicated to the project team via the patient's medical records.

Across the four medical wards, there are 156 inpatient beds, although occupancy was variable and generally not at capacity. The notes of every patient on the medical ward were reviewed; every patient who was present on the ward at the time of data collection was included.

Each round of data collection was carried out by one member of the team, consisting of two junior medical doctors and two physiotherapists, varying each week according to availability. At the beginning of the project, the study team designed the data collection tool as a group and piloted it together so that the tool was used consistently between team members. It was not possible for more than one team member to independently collect data each week. A uniform paper-based audit tool (see online supplementary figure 1) was used throughout the project, with subsequent analysis in spreadsheet software.

The project was grouped into two phases, with a handover to a different leader at week 14. During the first phase, data collection was planned to be weekly, and during the second phase data collection occurred 
when a member of the project team was available. This was for the pragmatic reason of attempting to make the data collection a less time-intensive and therefore a more sustainable task. Data were plotted as they were collected so that trends and associations with interventions could be observed and acted on during the project.

\section{DESIGN}

Since identification of and response to patients with TB spans multiple disciplines, it was important to establish a multidisciplinary team (MDT) to seek different experiences and perspectives. The team included IPC department members, physiotherapists, an infectious diseases specialist doctor and other medical doctors. TB Proof, a South African TB advocacy group, gave advice on strategies that had worked well in other South African settings. The project received support from the hospital's Chief Executive Officer (CEO) and Quality Assurance Director as the project evolved.

The ' 5 Whys' approach was used to conduct a root cause analysis. As discussed in the background, the main root causes were staffs' limited knowledge on TB infection control, suboptimal communication of a patient's infectiousness, insufficient isolation facilities, and stigma. The interventions focused on education: improving knowledge of when to apply IPC measures (which patients are potentially infectious) and how to apply these measures (including alternative isolation practices and correct use of face masks). Additionally, in order to improve communication of patients' infectiousness between staff, a supply of 'airborne precaution' signs were produced and distributed to the medical wards, along with training in their use.

Stakeholder discussions highlighted the need for a simple framework to help staff remember the key infection control measures. The acronym 'FISH'-'Facemask, Isolation, Sign, and ask for Help'-was developed. This formed the basis of the educational sessions.

Through informal discussions with MDT members, we anticipated problems gaining buy-in from medical staff including doctors and nurses due to conflicting demands on their time, and the perception that adhering to IPC practices is time-consuming and ineffective. We predicted stigma would be a major barrier to healthcare professionals asking patients to wear face masks, and that it would be difficult for patients to wear masks for extended periods of time. We anticipated that our intervention strategy would need to be tailored to different groups of healthcare professionals due to different experiences, knowledge and beliefs held by each group.

We worked to make the project sustainable by supporting the IPC nurses to perform regular audit as part of continuous quality improvement. We also worked to gain buy-in from senior managerial staff including the CEO and Quality Assurance Director so that the momentum for this project came from senior figures. Junior members of the team, such as junior physiotherapists, were mentored during the project to ensure that their skills were expanded in order to continue this work independently and to enable them to initiate similar projects in the future.

\section{STRATEGY}

\section{PDSA cycle 1 (with intervention at week 4)}

The aim of the first Plan-Do-Study-Act (PDSA) cycle was to improve knowledge of $\mathrm{TB}$, its spread and how IPC measures can be effectively applied to reduce transmission. The cycle comprised two core interventions. The first was ward-based teaching on the four medical wards led by the senior IPC Sister. This took place in a regular teaching slot at handover time, so night and day staff could attend. We felt the messages would be most successfully communicated by a fellow nurse who had a very good understanding of the problems on the ward. Teaching focused on general TB knowledge and provided guidance on the need for isolation. The second intervention was the development and distribution of the 'airborne precaution' signs by the IPC team. They ensured that each medical ward had a stock and knew when and how to use them.

We felt it was very important to have the buy-in and support from the IPC Sister. It became clear that teaching sessions would have to be frequent in order to embed new knowledge and to reach all members of the staff. Staff fed back that using the 'airborne precaution' sign and face masks was perceived by patients as stigmatising.

\section{PDSA cycle 2 (with intervention at week 7)}

During the next cycle we aimed to embed learning by introducing the FISH acronym, designed to help staff remember the key IPC interventions. The teaching during this cycle was inservice training, a seminar-style session which occurred outside of the ward environment. This took place in a well-equipped room with a projector, so we produced a PowerPoint presentation. At this session (and in the following week), flyers which explained the use of the FISH acronym were distributed around the wards and hospital.

It was positive that a large number of staff attended the teaching session. Student nurses, rather than senior Sisters, comprised the majority of audience; they were keen to learn but we found it difficult to influence senior members of the staff. As a result, we spent time with the Sisters on the ward following each round of data collection in order to feed back the results and discuss their concerns.

It became clear that N95 masks were stored in a locked cupboard out of access for the staff on the ward. We made efforts during this cycle to encourage Sisters to keep the masks in an unlocked communal area, but there was resistance to this because of concern they would be used inappropriately. We also encouraged other members of the staff to feel able to ask for N95 masks when required. 


\section{PDSA cycle 3 (with intervention at week 13)}

The aim of this cycle was to continue to improve staff's TB IPC knowledge in a fun and destigmatising way. There were several simultaneous interventions during this cycle, clustered together to form 'TB Awareness week'. A second inservice training session was organised which was attended by a representative of every department, including housekeeping, porters and catering staff. The TB project and its results to date were presented at this session, and further teaching around the FISH acronym given. More posters and flyers containing information about TB and infection control were distributed to staff and patients. A quiz competition was organised at the entrance to the hospital and on each of the medical wards. Members of the staff were asked questions relating to the FISH acronym and prizes were won for correct answers. A hospital-wide lecture, introduced by the CMH CEO, provided information about incidence and management of TB across the Eastern Cape and reinforced the FISH messages. Staff with personal experience of TB publicly shared their stories. Following the lecture, a cross-departmental competition of posters, songs and dramas conveying the key messages of the project were judged and prizes awarded.

This cycle was very effective at generating a feeling of excitement. The competitions were an engaging way of helping staff remember key IPC interventions. Having the public support of the CEO increased the credibility of the project.

\section{PDSA cycle 4 (with intervention at weeks 20, 21 and 22)}

Although improvement following cycle 3 was encouraging, we felt that further intervention was required to anchor change. This was the point at which the second phase of the project began with the aim of maintaining improvement. We felt that the strategies used during the previous three cycles had been successful, so during the fourth cycle we adopted a similar strategy to improve knowledge. Throughout the 3-week intervention, we:

- Held the quiz competition weekly as per cycle 3 .

- Hung more signs in outpatient and inpatient departments, reminding staff of the principles of TB IPC.

- Distributed information leaflets onto cars in the staff car park.

- Provided informal ad-hoc ward-based training for nurses.

- Provided formal in-service training for nurses.

In addition, we held an event on World TB Day (24 March 2017), where senior hospital and provincial government leaders spoke about the burden and challenges of $\mathrm{TB}$, with occupational TB survivors from the local area sharing their personal stories. Hospital and district staff joined local non-governmental organisations (NGOs) in a 'solidarity march' whereby we organised a procession to move from $\mathrm{CMH}$ to a nearby local TB hospital. The event was reported in the local newspaper and national television.
The World TB Day celebrations were time-intensive to plan and undertake but were felt by the team to be important at further raising the profile of the TB project within the hospital and community. By now, we felt staff's knowledge was improving (as the quizzes allowed us to test knowledge) and we felt that staff were becoming more comfortable talking about TB. This intervention had broader messages which targeted the whole hospital and community rather than just the medical wards, and as a result there was less focus on the IPC interventions on the medical wards. We felt the intervention was successful at improving morale and decreasing stigma, but attention moved away from ward-level IPC adherence.

\section{RESULTS}

Figure 1 shows the run chart of the three outcome measures over the 8-month period. Table 1 displays the compliance with each outcome measure per week for the duration of the project. The baseline mean compliance (the first 3 weeks) was 2\% (face mask use), 12\% (airborne precaution sign use) and 22\% (isolation practices).

In general, it appears that during and shortly after an intervention, adherence to each measure improved. This is particularly the case following the third intervention (week 13). Following initial improvement after this intervention, adherence waned until the fourth intervention, during which adherence again improved.

Following the fourth intervention (weeks 20, 21 and 22), during a time when active involvement with ward staff reduced in order to undertake a hospital-wide intervention, compliance decreased. The end-of-project post-intervention mean of the last three values is $6 \%$ (from 2\% for face masks), 21\% (from 12\% for signs) and $57 \%$ (from 22\% for isolation). It is possible that this may in part be due to the fact that there were fewer patients on the ward who required IPC measures during the second phase of the project (i.e., the numbers are smaller). A further reason may be that new medical wards were soon to open with better infection control facilities. Staff's enthusiasm for improving practice on their current wards may have decreased as their focus turned to anticipating moving to a new clinical environment.

The improvement in adherence is different for each outcome measure. Isolation has had the greatest improvement in compliance, whereas improvement in face mask usage has not been sustained from baseline. We believe that the degrees of stigma associated with each intervention are the main cause of the differences in improvement. We increasingly understood that the face mask was thought to be synonymous with TB infection, and despite recurrent explanations that both masks and the signs were necessary for any airborne disease, nursing staff continued to report that they perceived the use of a face mask in particular as highly stigmatising. In contrast, isolation was required for many, often less stigmatising conditions, and therefore nurses reported less reluctance to do this. 


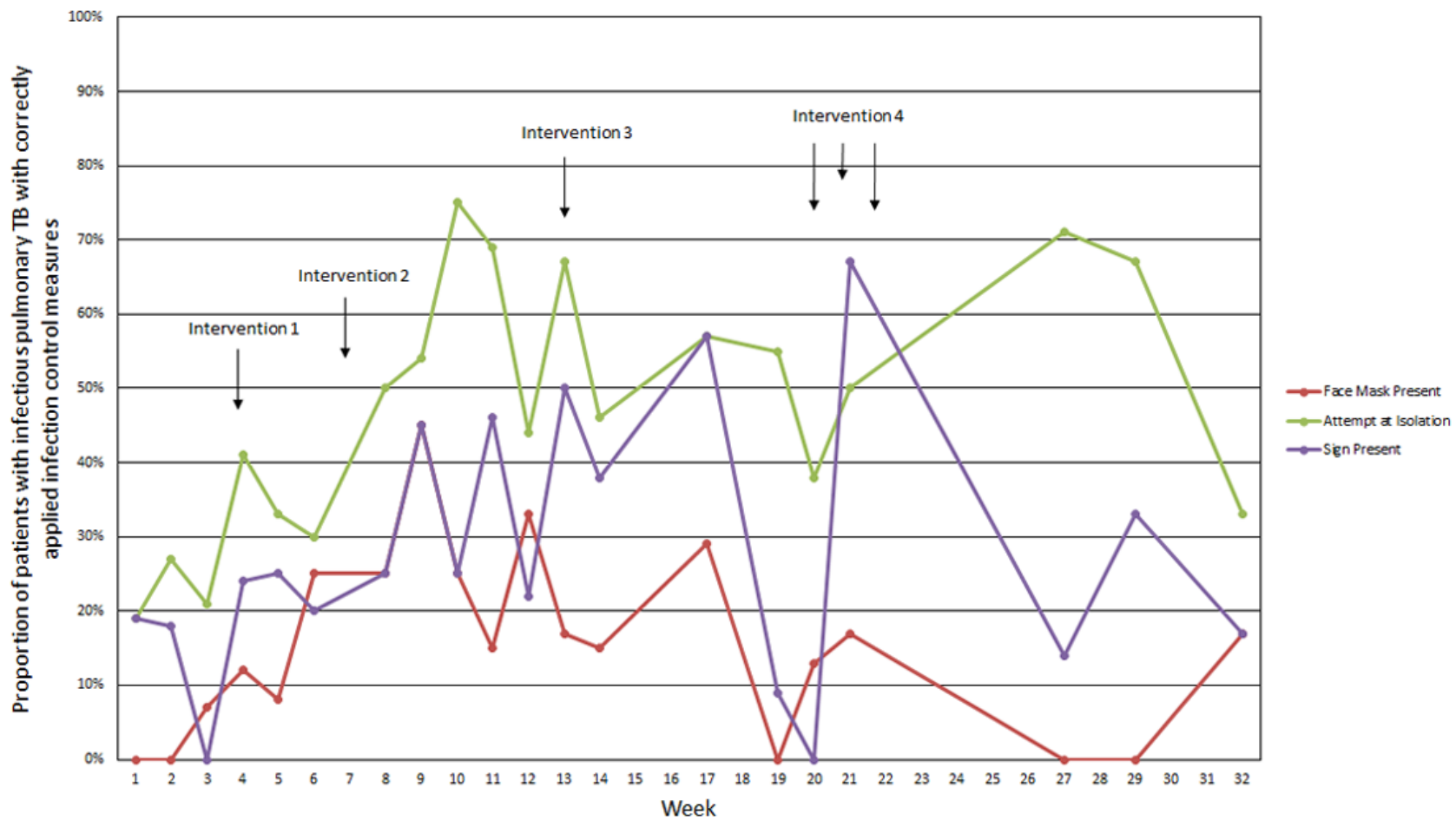

Figure 1 Run chart showing adherence to infection control measures over 32 weeks. TB, tuberculosis.

Table 1 Proportion of patients with infectious pulmonary tuberculosis with correctly applied infection control measures

\begin{tabular}{llll}
\hline Week & $\begin{array}{l}\text { Face mask } \\
\text { present, \% (n) }\end{array}$ & $\begin{array}{l}\text { Isolated, } \% \\
\text { (n) }\end{array}$ & $\begin{array}{l}\text { Sign present, } \\
\%(\mathbf{n})\end{array}$ \\
\hline 1 & $0(0 / 16)$ & $19(3 / 16)$ & $19(3 / 16)$ \\
\hline 2 & $0(0 / 11)$ & $27(3 / 11)$ & $18(2 / 11)$ \\
\hline 3 & $7(1 / 14)$ & $21(3 / 14)$ & $0(0 / 14)$ \\
\hline 4 & $12(2 / 17)$ & $41(7 / 17)$ & $24(4 / 17)$ \\
\hline 5 & $8(1 / 12)$ & $33(4 / 12)$ & $25(3 / 12)$ \\
\hline 6 & $25(5 / 20)$ & $30(6 / 20)$ & $20(4 / 20)$ \\
\hline 8 & $25(2 / 8)$ & $50(4 / 8)$ & $25(4 / 8)$ \\
\hline 9 & $45(5 / 11)$ & $54(6 / 11)$ & $45(5 / 11)$ \\
\hline 10 & $25(5 / 20)$ & $75(15 / 20)$ & $25(5 / 20)$ \\
\hline 11 & $15(2 / 13)$ & $69(9 / 13)$ & $46(6 / 13)$ \\
\hline 12 & $33(3 / 9)$ & $44(4 / 9)$ & $22(2 / 9)$ \\
\hline 14 & $17(1 / 6)$ & $67(4 / 6)$ & $50(3 / 6)$ \\
\hline 17 & $15(2 / 13)$ & $46(6 / 13)$ & $38(5 / 13)$ \\
\hline 19 & $29(2 / 7)$ & $57(4 / 7)$ & $57(4 / 7)$ \\
\hline 20 & $0(0 / 11)$ & $55(6 / 11)$ & $9(1 / 11)$ \\
\hline 21 & $13(1 / 8)$ & $38(3 / 8)$ & $0(0 / 8)$ \\
\hline 27 & $17(1 / 6)$ & $50(3 / 6)$ & $67(4 / 6)$ \\
\hline 29 & $0(0 / 7)$ & $71(5 / 7)$ & $4(1 / 7)$ \\
\hline 32 & $17(1 / 7)$ & $67(2 / 3)$ & $33(1 / 3)$ \\
\hline & & $33(2 / 6)$ & $17(1 / 6)$ \\
\hline
\end{tabular}

It is not possible to confidently determine whether interventions were responsible for changes in IPC adherence. This is because each intervention had multiple components. The period of time between interventions was short which makes it difficult to determine which intervention was taking effect.

Overall, change does not yet appear to be stable, as indicated by the variable proportion of adherence from week to week.

\section{LESSONS AND LIMITATIONS \\ Strengths of the project}

- The goals of the project were consistent throughout and were in line with national IPC policy. This was important for credibility of our messages.

- The approach adopted is likely to be generalisable to other hospitals within SA. This is because the problems encountered are not unique to $\mathrm{CMH}$ and the techniques adopted are resource-light and do not require specialist equipment. Materials such as the audit tool, training materials and posters can be shared with other hospitals in a TB IPC 'toolkit'.

- The project team was multidisciplinary, which was important in order to achieve a thorough and accurate understanding of the root causes of the problems, important for achieving sustainability of improvement.

- We adopted different educational styles to suit different groups of people. Interventions were fun 
and engaging and provided educational opportunities distinct from didactic teaching.

- The awareness week and World TB Day brought together people from different backgrounds and provided a sense of unity, of belonging to a wider community and of seeing the 'bigger picture', each important for inspiring positive, 'can-do' attitudes. Multiple organisations who were previously unconnected worked together and these links are now established for future working relationships. Although these outcomes of the project were not being measured, these represent significant positive gains that may increase the likelihood of sustainable improvements.

\section{Limitations of methodology}

- Data collection during the second phase of the project was convenience-based.

- There were four different data collectors during the project who were each trained to collect data in the same manner; however, interuser variability was not measured.

- Each cycle of improvement included multiple simultaneous interventions; therefore, it is not possible to determine the effect of a single individual intervention on IPC adherence. Conclusions can only be drawn regarding the groups of interventions.

\section{Problems encountered}

- We underestimated the power of stigma as a barrier to engaging with IPC practices, particularly face mask use which showed the lowest improvement in compliance. Nursing staff reported feeling worried about asking patients to wear face masks or moving patients into isolation for fear they would be accused of being discriminatory. We feel this is the primary reason why our improvements were not sustained, as this requires constant reassurance, encouragement and time to overcome.

- We aimed to recruit a 'TB Ambassador' on each ward whose role would be to ensure compliance with practices and to monitor stock of 'airborne precaution' signs and face masks. We asked ambassadors to come forward voluntarily but there was little support for this from ward nurses.

- It emerged that it was important for nurses to know that what we were teaching was hospital policy and part of a standard operating procedure (SOP). There was resistance to accept the change in practice until this was formalised.

- Both the TB awareness week (intervention 3) and World TB Day events (intervention 4) were intensive interventions which targeted the whole hospital, including administration staff, patients and the community. While this spread the messages further than the targeted medical wards, it meant our efforts were not focused on the medical wards and is possibly the reason the intervention did not make as big an improvement as hoped. However, the involvement of other departments of the hospital (which we were not measuring) may result in improved practice beyond the medical wards.

\section{Lessons learnt}

- We found that only a small proportion of nurses attended training away from the wards (due to difficulty leaving their ward duties). Those who attended were junior nurses or nursing students. We therefore gained little buy-in from senior nurses, and as a result their influence over junior nurses was limited. In future, in-service training sessions should be primarily ward-based, rather than classroom-based, which should improve attendance.

- We felt that our interventions were less effective at influencing senior nurses to change their practice. In future, we would spend more time one-on-one with senior nurses to understand their perspective and gain their buy-in before commencing the project.

- It became clear that a patient's TB status was not being communicated to nurses from doctors. In future, more emphasis should be placed on this communication link and facilitating a team-working environment between the two professions.

\section{Recommendations for future initiatives}

- Project team: We recommend developing a project team comprising the medical matron, a lead physician, the infection control team, and members of the wider clinical team and hospital staff. Gaining formal approval from the hospital directorate is important to achieve prior to commencing the project. They should be responsible for distributing initial information to the ward teams to demonstrate senior buy-in. We recommend exploring the potential for partnership between local NGOs and other hospitals in order to share resources and experiences. A 'TB Ambassador' for each ward should be selected.

- Project design: An initial phase of the project should aim to understand the cultural beliefs and knowledge of patients and staff. This could take the form of one-to-one interviews or focus groups with core stakeholders. We recommend having separate sessions for each professional group, as in our experience views differ significantly.

- The team should then focus on designing and implementing interventions which target common misconceptions identified. These interventions should aim to improve the level of understanding of TB, its transmission, and the role of face masks and isolation in infection control. We recommend the use of narratives and story-telling by those with personal experience of TB, particularly staff members, as we felt this was effective at helping staff understand that they are at risk of TB infection.

- The team should design an SOP, outlining the expected changes to practice and the audit process. 
This should be approved by the hospital directorate before attempting to change practice on the ward.

- We recommend ward-based introduction of the SOP on a minimum of three occasions by senior members of nursing staff. This could then be supplemented with mandatory in-service training and hospital-wide awareness events (as part of individual PDSA cycles), aiming to reinforce understanding of the roles of staff and to challenge any ongoing misconceptions.

- Maintaining momentum: We recommend data are collected every other week. During the maintenance phase of the project, this could be carried about by the infection control team on a quarterly basis, forming part of their routine audit work. We recommend measuring staff's knowledge of TB IPC in addition to compliance with infection control measures. We recommend giving 'real-time' feedback, by immediately discussing the results of each audit with the member of staff caring for the patient and the nurse in charge. Displaying up-to-date run charts on each ward allows progress to be visualised, and cross-ward comparison at departmental meetings may encourage healthy competition.

\section{CONCLUSION}

Ensuring sustainable improvements in practice is a particular challenge for TB, a disease that is 'invisible'. Personal risk appears abstract and infection control perceived as burdensome. A further barrier to compliance is stigma; working against stigma is not simply an institutional issue, as it manifests into the local community and beyond. We have learnt that to overcome these barriers, intensive and persistent intervention is necessary on the ward, targeting front-line staff but also within the hospital leadership, because change must become institutional culture.

Throughout the project, methods to ensure sustainability have been our primary concern. The principal way we attempted to achieve this was by forming an MDT which worked with existing hospital structures, rather than in parallel to them. We worked to gain the buy-in from senior managerial staff so that momentum for change was led by them.

It is clear from our data that further work is required to ensure adherence to TB IPC is sustainable and stable. The next stage of the project is to work with the hospital's TB IPC officer to produce a hospital TB IPC plan and SOP. We hope this will encourage adherence to policy and improve accountability for inaction, and ensure continual monitoring and evaluation of IPC practices as part of routine IPC work.

Acknowledgements We would like to thank the staff of Cecilia Makiwane Hospital for their support and assistance with this project, in particular Dr Stead, the infection prevention control team and the physiotherapy team (with special thanks to Eden Smith and Siphosethu Maqwelane). We would also like to thank our external collaborators (Nkqubela TB Hospital, TB Proof, Beyond Zero and Treatment Action Campaign).

Contributors Conceived and designed the project: FK, VG, ILH, AP. Undertook the quality improvement work: FK, VG, ILH. Analysed and interpreted the data: FK, ILH, AP. Wrote the paper: ILH, FK, VG, AP. All authors read and approved the final manuscript. ILH, FK and AP were responsible for the overall content as guarantors.

Funding This project was undertaken during ILH's and FK's Improving Global Health through Leadership Development placements, supported by the NHS Thames Valley and Wessex Leadership Academy. No specific funding was received for the project.

Competing interests None declared.

Patient consent for publication Not required.

Ethics approval Ethical approval for this project was obtained from the local ethics review board prior to the collection of data.

Provenance and peer review Not commissioned; externally peer reviewed.

Open access This is an open access article distributed in accordance with the Creative Commons Attribution Non Commercial (CC BY-NC 4.0) license, which permits others to distribute, remix, adapt, build upon this work non-commercially, and license their derivative works on different terms, provided the original work is properly cited, appropriate credit is given, any changes made indicated, and the use is non-commercial. See: http://creativecommons.org/licenses/by-nc/4.0/.

\section{REFERENCES}

1. World Health Organization. Global Tuberculosis report 2016: Country profiles. World Health Organization 2016.

2. Statistics South Africa. Mortality and causes of death in South Africa: Findings from death notification. 2015;2014.

3. National Department of Health South Africa. National Department of Health Annual performance plan 2014/15 - 2016/17. 2014.

4. Company UR. Tuberculosis in Healthcare Workers : Findings from South Africa. Infection Control 2008.

5. World Health Organization. The End TB Strategy. World Health Organization 2015.

6. Statistics South Africa. Population of Mdantsane - Census 2011 [Internet]. $2011 \mathrm{https}: / /$ census2011.adrianfrith.com/place/260088

7. Statistics South Africa. Mid-year population estimates. 2014.

8. Shah NS, Auld SC, Brust JC, et al. Transmission of Extensively Drug-Resistant Tuberculosis in South Africa. N Engl J Med 2017;376:243-53.

9. World Health Organization. WHO Policy on TB infection control in health-care facilities. World Health Organization, 2009.

10. Jensen PA, Lambert LA, lademarco MF, et al. Guidelines for preventing the transmission of Mycobacterium tuberculosis in healthcare settings, 2005. MMWR Recomm Rep 2005;54:1-141.

11. Department of Health South Africa. National Infection, Prevention and Control Policy for TB, MDRTB and XDRTB. 2007.

12. Sissolak D, Marais F, Mehtar S. TB infection prevention and control experiences of South African nurses-a phenomenological study. BMC Public Health 2011;11:1-10. 